\section{Revising Regularization with Linear Approximation Term for Compressive Sensing Improvement}

\section{Zan Chen, Xingsong Hou, Ling Shao, Shidong Wang}

In this letter, we propose a novel revised regularization to improve the performance of compressive sensing (CS) reconstruction. We suppose that a specific regularization term is insufficient to accommodate the prior information of CS while it can be improved by further imposing a linear approximation term. We also prove that the revised regularization is substantially equivalent to the CS preprocessing methods. We conduct extensive experiments on various CS algorithms which show the effectiveness of our revised regularization.

Introduction: Compressive sensing (CS) theory has been widely employed in various applications, such as image coding and transmission [1]. CS theory permits that using a certain regularization, the original signal can be reconstructed accurately from a small set of measurements

$$
y=\Phi x
$$

where $\Phi \in R^{m \times n}(m<n)$ is a CS measurement matrix which satisfies restricted isometry property (RIP), $x \in R^{n}$ is the image, and $y \in R^{m}$ is the observation vector. Since $\Phi$ is rank-deficient, it requires to solve the ill-posed CS reconstruction problem by introducing some additional regularization information. By selecting an appropriate regularization parameter $\lambda$, CS enable to reconstruct an image $x$ from $y$ by solving the following unconstrained optimization problem:

$$
x=\arg \min _{x}\|\Phi x-y\|_{2}^{2}+\lambda G(x),
$$

where $G(x)$ is a regularization function that introduces prior information on the expected solution. The popular regularization terms include $l_{1}$ norm, total variation norm, and non-local rank-norm. The recent adaptive data-driven regularization, which can be learned according to the datasets by using deep neural, has become a more efficient way to the inverse problem [2]. The CS reconstruction performance is closely associated with the selection of the regularization term.

In this letter, we propose a revised regularization method based on the linear approximation to improve the CS reconstruction performance effectively. We provide sufficient evidence to show that the proposed regularization term is a generalization of the preprocessing method for CS reconstruction improvement. We analysis that the sparsefiltering preprocessing method [3] and collaboration reduced rank preprocessing method [4] are special cases, which adopt the proposed linear approximation revised regularization for wavelet sparse prior and low-rank prior respectively. We further test the improvement of CS reconstruction by applying the proposed method to the state-of-the-art data-driven regularization [5]. The experimental results show that the proposed revised regularization term can significantly improve the CS reconstruction performance.

Solving method for general regularization: Eq. 2 can be solved by introducing an auxiliary variable $z$ that is constrained to be equal to $x$, which gives us the following optimization problem [6]:

$$
(x, z)=\arg \min _{x, z}\|\Phi x-y\|_{2}^{2}+\lambda G(z)+\mu\|z-x\|_{2}^{2} .
$$

Eq. 3 can be solved analytically over $x$ and $z$ in two alternating steps, which consists of the following iterations [6]:

$$
\begin{aligned}
& x^{k}=\arg \min _{x}\|\Phi x-y\|_{2}^{2}+\mu\left\|z^{k-1}-x\right\|_{2}^{2}, \\
& z^{k}=\arg \min _{z}\left\|z-\left(x^{k}-\lambda \nabla_{z} G\left(x^{k}\right)\right)\right\|_{2}^{2}+\mu\left\|z-x^{k}\right\|_{2}^{2},
\end{aligned}
$$

where $\nabla_{z} G\left(x^{k}\right)$ is the gradient descent of $G$. In the first step Eq. 4, a strictly quadratic convex function has to be minimized, which leads to the following linear formulation:

$$
x^{k}=\left(\Phi^{T} \Phi+\mu I\right)^{-1}\left(\Phi^{T} y+\mu z^{k-1}\right) .
$$

In the second step, setting the gradient of Eq. 5 to be zero, we can acquire the closed-form solution to $z$ sub-problem as follows

$$
z^{k}=\frac{\mu x^{k}+\left(x^{k}-\lambda \nabla_{z} G\left(x^{k}\right)\right)}{\mu+1}=x^{k}-\rho \nabla_{z} G\left(x^{k}\right),
$$

where $\rho=\frac{\lambda}{\mu+1}$. By iterating Eq. 6 and Eq. 7, CS can output the final reconstructed image. This two-step splitting approach is the basic framework for CS reconstruction algorithm, which we can use to deal with different kinds of CS regularization.

Revising regularization with linear approximation: In addition to the generally certain regularization term, we additionally assume that $\left(\Phi^{T} \Phi z\right)$ also needs to satisfy the prior assumption. Suppose $\bar{x}$ is the original image. Then the linear approximation of the regularization term $G\left(\Phi^{T} \Phi z\right)$ at the point of $\bar{x}$ can be formulated as

$$
G\left(\Phi^{T} \Phi z\right) \approx G(\bar{x})+\left(\Phi^{T} \Phi z-\bar{x}\right)^{T} \nabla_{z} G(\bar{x}) .
$$

By further imposing linear approximation regularization term to the original one, our novel revised regularization term can be written as

$$
\begin{aligned}
R(z) & =G(z)+G\left(\Phi \Phi^{T} z\right) \\
& =G(z)+G(\bar{x})+\left(\Phi^{T} \Phi z-\bar{x}\right)^{T} \nabla_{z} G(\bar{x}),
\end{aligned}
$$

which has

$$
\nabla_{z} R(z)=\nabla_{z} G(z)+\Phi^{T} \Phi \nabla_{z} G(\bar{x}) .
$$

Replacing the regularization term $G$ in Eq. 3 with the new revised term $R$, the two-step iterative solving process should be represented as

$$
\begin{aligned}
& x^{k}=\left(\Phi^{T} \Phi+\mu I\right)^{-1}\left(\Phi^{T} y+\mu z^{k-1}\right), \\
& z^{k}=x^{k}-\rho \nabla_{z} R\left(x^{k}\right)=x^{k}-\rho \nabla_{z} G\left(x^{k}\right)-\rho \Phi^{T} \Phi \nabla_{z} G(\bar{x}),
\end{aligned}
$$

In the iteration process for Eq. 11 and Eq. 12, we can move the third term $\rho \Phi^{T} \Phi \nabla_{z} G(\bar{x})$ in Eq. 12 to Eq. 11, which does not change the final iterative calculation result. Thus, Eq. 11 and Eq. 12 can be further rewritten as

$$
\begin{aligned}
x^{k} & =\left(\Phi^{T} \Phi+\mu I\right)^{-1}\left(\Phi^{T} y+\mu z^{k-1}-\mu \rho \Phi^{T} \Phi \nabla_{z} G(\bar{x})\right), \\
z^{k} & =x^{k}-\rho \nabla_{z} G\left(x^{k}\right)
\end{aligned}
$$

Considering $y=\Phi \bar{x}$, Eq. 13 can be further formulated as

$$
x^{k}=\left(\Phi^{T} \Phi+\mu I\right)^{-1}\left(\Phi^{T} \tilde{y}+\mu z^{k-1}\right),
$$

where $\tilde{y}$ is measured from the preprocessing image $\left(\bar{x}-\mu \rho \nabla_{z} G(\bar{x})\right)$ :

$$
\tilde{y}=\Phi\left(\bar{x}-\mu \rho \nabla_{z} G(\bar{x})\right) .
$$

Thus, we can use $\tilde{y}$ instead of $y$ to reconstruct the original image. It is not difficult to observe that our revised CS regularization term is equivalent to go through CS preprocessing.

Preprocessing for CS improvement: Fig. 1 shows the block diagram of the CS preprocessing method, where $\left(\bar{x}-\mu \rho \nabla_{z} G(\bar{x})\right)$ represents the relevant preprocessing for different regularization $G$. Note that the process of $\left(\bar{x}-\mu \rho \nabla_{z} G(\bar{x})\right)$ can be seen as the vertical and horizontal finite difference value thresholding operator, wavelet value thresholding operator, singular value thresholding operator, and denoising convolution neural network for TVNL [7], TSW [8], NLR [9] and DLAMP [5], respectively.

The sparse-filtering preprocessing method [3] cuts off the small wavelet coefficients before CS measurement, which is a preprocessing method using wavelet value thresholding operator. Also, collaboration reduced rank preprocessing method [4] strengthens the non-local sparsity using low-rank approximation before CS measurement, which is a preprocessing method using singular value thresholding operator. Both methods can significantly improve CS reconstructed PSNR results using preprocessing, and they are just the individual cases of the proposed linear approximation revised regularization. With our revised regularization, we can easily extend the CS preprocessing method to the more advanced CS reconstruction algorithm, such as LDAMP [5]. 
Original
Image $\bar{x}$$\rightarrow \stackrel{\begin{array}{c}\text { Preprocessing } \\ \tilde{x}=\bar{x}-\mu \rho \nabla_{z} G(\bar{x})\end{array}}{\rightarrow} \rightarrow \begin{gathered}\text { CS measurement } \\ \tilde{y}=\Phi \tilde{x}\end{gathered} \rightarrow$ CS reconstruction $\rightarrow \begin{gathered}\text { Recovery } \\ \text { Image }\end{gathered}$

Fig. 1. The block diagram of CS preprocessing scheme.

Experimental results and discussion: The experimental results are based on four classical CS reconstruction algorithms: TVNL-CS [7], TSW-CS [8], NLR-CS [9], and LDAMP-CS [5], which use total variation based non-local regularization, tree-structure wavelet sparse regularization, non-local low-rank regularization, and learned data-driven based regularization, respectively. The relevant parameters, including $\mu, \lambda$ and $\rho$, are set following the original papers. We compare the reconstructed results for the original regularization and the corresponding revised regularization. We test four standard $256 \times 256$ size gray-scale images, including Barbara, Lena, Peppers, and Cameraman, to evaluate the performance of the proposed method.

Table 1 presents the peak signal-to-noise ratio (PSNR) results between the original image and the CS reconstructed image for different algorithms. 'LAR' refers to the proposed linear approximation revised regularization method. The higher PSNR values between the original regularization and the revised one are highlighted in bold in each cell We can see that our linear approximation revised regularization can significantly improve the CS reconstruction performance of the original regularization. The proposed LAR-LDAMP can achieve the state-of-theart CS reconstruction results.

Fig. 2 and Fig. 3 illustrate the reconstructed Lena using the original regularization and the revised regularization at sampling ratio 0.1 respectively. We can see that the proposed algorithm can achieve a much better visual effect.

Table 1: Reconstructed PSNRs for different algorithms

\begin{tabular}{|c|c|c|c|c|c|c|}
\hline \multirow{2}{*}{ Images } & \multirow{2}{*}{ Methods } & \multicolumn{5}{|c|}{ Sampling ratios } \\
\hline & & 0.1 & 0.2 & 0.3 & 0.4 & 0.5 \\
\hline \multirow{8}{*}{ Barbara } & TVNL & 23.16 & 25.77 & 28.24 & 31.39 & 33.74 \\
\hline & LAR-TVNL & 23.74 & 26.19 & 29.11 & 31.78 & 33.96 \\
\hline & TSW & 25.10 & 27.73 & 30.18 & 32.84 & 34.77 \\
\hline & LAR-TSW & 25.44 & 28.67 & 35.79 & 37.57 & 36.86 \\
\hline & NLR & 29.43 & 34.56 & 35.18 & 39.45 & 41.43 \\
\hline & LAR-NLR & 30.94 & 36.51 & 37.70 & 41.61 & 42.95 \\
\hline & LDAMP & 27.39 & 32.74 & 36.01 & 37.95 & 39.36 \\
\hline & LAR-LDAMP & 28.08 & 34.29 & 37.34 & 39.40 & 40.79 \\
\hline \multirow{8}{*}{ Lena } & TVNL & 27.18 & 30.76 & 32.75 & 35.54 & 36.56 \\
\hline & LAR-TVNL & 27.64 & 31.38 & 34.24 & 36.25 & 37.90 \\
\hline & TSW & 26.98 & 30.89 & 33.52 & 36.17 & 38.09 \\
\hline & LAS-TSW & 29.87 & 34.95 & 38.73 & 41.80 & 41.34 \\
\hline & NLR & 31.26 & 35.85 & 38.39 & 40.93 & 42.73 \\
\hline & LAR-NLR & 32.22 & 37.17 & 40.39 & 42.24 & 43.50 \\
\hline & LDAMP & 32.04 & 36.39 & 39.26 & 41.24 & 42.90 \\
\hline & LAR-LDAMP & 33.10 & 37.08 & 39.65 & 41.77 & 43.55 \\
\hline \multirow{8}{*}{ Peppers } & TVNL & 26.96 & 31.16 & 32.97 & 35.67 & 36.09 \\
\hline & LAR-TVNL & 27.07 & 31.73 & 34.19 & 36.02 & 37.21 \\
\hline & TSW & 24.20 & 27.11 & 31.66 & 33.74 & 35.26 \\
\hline & LAR-TSW & 24.36 & 28.64 & 36.64 & 39.28 & 37.44 \\
\hline & NLR & 30.50 & 34.04 & 35.65 & 37.56 & 39.11 \\
\hline & LAR-NLR & 31.86 & 35.94 & 37.92 & 39.58 & 40.59 \\
\hline & LDAMP & 31.04 & 34.38 & 36.56 & 38.01 & 39.27 \\
\hline & LAR-LDAMP & 32.86 & 35.69 & 38.09 & 39.52 & 40.55 \\
\hline \multirow{8}{*}{ Cameraman } & TVNL & 25.66 & 28.74 & 31.07 & 33.51 & 36.60 \\
\hline & LAR-TVNL & 26.32 & 29.77 & 32.08 & 34.34 & 36.61 \\
\hline & TSW & 23.58 & 26.25 & 29.59 & 32.06 & 34.81 \\
\hline & LAR-TSW & 24.10 & 28.71 & 35.35 & 36.89 & 38.59 \\
\hline & NLR & 27.30 & 31.67 & 34.14 & 36.83 & 38.24 \\
\hline & LAR-NLR & 28.40 & 32.71 & 35.49 & 38.25 & 38.92 \\
\hline & LDAMP & 29.29 & 32.17 & 35.60 & 37.50 & 38.80 \\
\hline & LAR-LDAMP & 30.90 & 33.83 & 36.43 & 38.57 & 40.00 \\
\hline \multirow{8}{*}{ Average } & TVNLM & 25.74 & 29.11 & 31.26 & 34.03 & 35.75 \\
\hline & TVNLM-LAS & 26.19 & 29.77 & 32.41 & 34.60 & 36.42 \\
\hline & TSW & 24.97 & 28.00 & 31.24 & 33.70 & 35.73 \\
\hline & TSW-LAS & 25.94 & 30.24 & 36.63 & 38.89 & 38.56 \\
\hline & NLR & 29.62 & 34.03 & 35.84 & 38.69 & 40.38 \\
\hline & NLR-LAS & 30.86 & 35.58 & 37.88 & 40.42 & 41.49 \\
\hline & LDAMP & 29.94 & 33.92 & 36.86 & 38.68 & 40.08 \\
\hline & LDAMP-LAS & 31.24 & 35.22 & 37.88 & 39.82 & 41.22 \\
\hline
\end{tabular}

Conclusion: In this work, we propose a novel revised regularization with linear approximation term for CS reconstruction improvement. We also prove that the proposed regularization is substantially similar to CS preprocessing. We further test the proposed method with different kinds of CS regularization. The experimental results show that the proposed

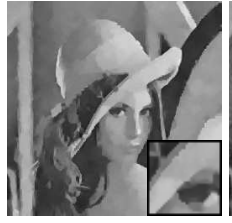

$27.18 \mathrm{~dB}$ (a) TVNL

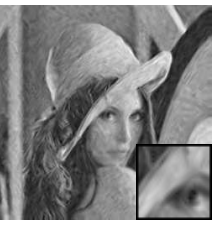

$26.98 \mathrm{~dB}$

(b) $T S W$

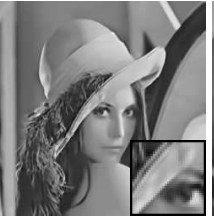

$31.26 \mathrm{~dB}$

(c) NLR

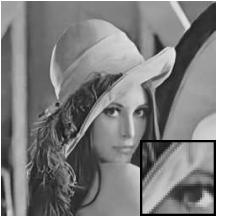

$32.04 \mathrm{~dB}$

(d) $L D A M P$
Fig. 2. Using original regularization terms at sampling ratio 0.1 .

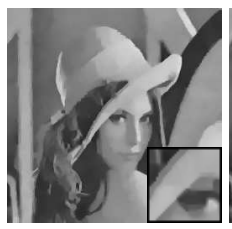

$27.64 \mathrm{~dB}$ (a) LAR-TVNL

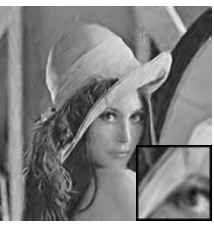

$29.87 \mathrm{~dB}$

(b) LAR-TSW

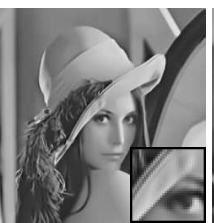

$32.22 \mathrm{~dB}$

(c) $L A R-N L R$

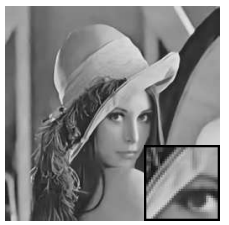

$33.10 \mathrm{~dB}$
Fig. 3. Using the revised regularization terms at sampling ratio 0.1 .

revised regularization can significantly improve the CS reconstruction performance compared to the original regularization.

Acknowledgment: This work was supported by National Key R\&D Program of China (2017YFF0107700), NSFC (61872286, u1531141, 61701391), and Guangdong Provincial Science and Technology Plan Project (2017A010101006, 2017B010123003).

Zan Chen and Xingsong Hou (School of Electronic and Information Engineering, Xi'an Jiaotong University, Xi'an 710049, PRC)

Ling Shao (Inception Institute of Artificial Intelligence, Abu Dhabi, United Arab Emirates)

Shidong Wang (School of Computing Sciences, University of East Anglia, Norwich, UK)

E-mail: houxs@mail.xjtu.edu.cn

Xingsong Hou: also with Guangdong Xi'an Jiaotong University Academy, PRC

\section{References}

1 Z. Chen, X. Hou, X. Qian, and C. Gong, "Efficient and robust image coding and transmission based on scrambled block compressive sensing," IEEE Transactions on Multimedia, vol. 20, no. 7, pp. 1610-1621, 2018.

2 T. Meinhardt, M. Möller, C. Hazirbas, and D. Cremers, "Learning proximal operators: Using denoising networks for regularizing inverse imaging problems," in IEEE International Conference on Computer Vision, 2017, pp. 1781-1790.

3 X. Hou, L. Zhang, Z. Chen, and C. Gong, "Sparse-filtering in directional lifting wavelet transform domain based bayesian compressive sensing," International Journal of Wavelets Multiresolution \& Information Processing, vol. 12, no. 06, p. 1450043, 2014.

4 Y. Tan, X. Hou, Z. Chen, and S. Yu, "Image compressive sensing reconstruction based on collaboration reduced rank preprocessing," Electronics Letters, vol. 53, no. 11, pp. 717-718, 2017.

5 C. Metzler, A. Mousavi, and R. Baraniuk, "Learned d-amp: Principled neural network based compressive image recovery," in Advances in Neural Information Processing Systems, 2017, pp. 1772-1783.

6 M. V. Afonso, J. M. Bioucas-Dias, and M. A. Figueiredo, "Fast image recovery using variable splitting and constrained optimization," IEEE transactions on image processing, vol. 19, no. 9, pp. 2345-2356, 2010.

7 J. Zhang, S. Liu, R. Xiong, S. Ma, and D. Zhao, "Improved total variation based image compressive sensing recovery by nonlocal regularization," in Circuits and Systems (ISCAS), 2013 IEEE International Symposium on, 2013, pp. 2836-2839.

8 L. Qin, J. Tan, Z. Wang, G. Wang, and X. Guo, "Exploiting the treestructured compressive sensing of wavelet coefficients via block sparse bayesian learning," Electronics Letters, vol. 54, no. 16, pp. 975-976, 2018.

9 W. Dong, G. Shi, X. Li, Y. Ma, and F. Huang, "Compressive sensing via nonlocal low-rank regularization," IEEE Transactions on Image Processing, vol. 23, no. 8, pp. 3618-3632, 2014. 